\title{
Acta
Biochimica
Polonica
}

Vol. 48 No. $3 / 2001$

$755-762$

QUARTERLY

\section{Effect of tartaric acid on conformation and stability of human prostatic phosphatase: An infrared spectroscopic and calorimetric study}

\author{
Sławomir Bem and Włodzimierz S. Ostrowski ${ }^{\square}$ \\ Institute of Medical Biochemistry, Collegium Medicum, Jagiellonian University, Kraków, Poland
}

Received: 23 April, 2001; revised: 16 May, 2001; accepted: 29 August, 2001

Key words: acid phosphatase, prostate, tartrate, secondary structure

\begin{abstract}
The solution structure and thermal stability of human prostatic acid phosphatase (hPAP) in the absence and in the presence of tartaric acid were studied by Fourier transform infrared spectroscopy (FTIR) and differential scanning calorimetry (DSC). The temperature dependence of the infrared spectrum and DSC scans indicate that hPAP undergoes thermal unfolding at a temperature between 49.5 and $52.5^{\circ} \mathrm{C}$. Binding of tartaric acid does not lead to major changes in the secondary structure of hPAP, however, hPAP with bound tartaric acid shows a significantly increased thermal stability. These results helped to better understand the mechanism of hPAP unfolding at the elevated temperature.
\end{abstract}

For more than fifty years, human prostatic acid phosphatase (hPAP) (EC 3.1.3.2) has been measured in blood serum for diagnosis and staging of prostatic tumors, however, the physiological role of this enzyme remains unknown. Therefore there is a need for further studies of the enzyme's properties in order to fully understand its biological function and catalytic mechanism.

The inhibition of hPAP by derivatives of tartaric acid has been investigated since 1957 when Kilsheimer \& Axelrod [1] have observed effective, competitive inhibition of the enzyme by the parent L(+)-tartrate. The latter has $K_{\mathrm{i}}=2.9 \times 10^{-5} \mathrm{M}$ at pH 5.0 and its ability to inhibit hPAP is of stereospecific nature [2]. The inhibitor must possess a hydroxyl group of $\mathrm{D}$ configuration in the $\alpha$-position and the $\beta$-carbon must be part of a carboxyl group or be attached to a carboxyl or hydroxyl group [1]. The strong interaction observed between $\mathrm{L}(+)$-tartrate and hPAP was later routinely used in affinity chromatography for prostatic phosphatase purification [3].

\footnotetext{
Institute of Medical Biochemistry, Collegium Medicum, Jagiellonian University, M. Kopernika 7, 31-034 Kraków, Poland; fax: (48 12) 422 2791, tel.: (48 12) 422 3272; e-mail: ostrowski@zdp.pan.krakow.pl Abbreviations: DSC, differential scanning calorimetry; FTIR, Fourier transform infrared spectroscopy; hPAP, human prostatic acid phosphatase.
} 
X-Ray crystal structure of hPAP has been determined at $3.1 \AA$ resolution [4] and of hPAP complexed with $\mathrm{L}(+)$-tartrate ions at $2.9 \AA$ resolution [2]. Binding of the inhibitor to the active site of the enzyme is due to the electrostatic interaction between the enzyme and the anionic ligand [5] and the extensive hydrogen bonding between hydroxyl group of the tartrate ion and the enzyme molecule [2]. The latter interactions involve a large part of the active site, which is located in an open cleft and appears as a doughnut shaped structure between the two domains [6]. Only side chain atoms are involved in L(+)-tartrate binding [2, 5]. Upon the inhibitor binding hPAP does not experience a significant structural change. Most of this change occurs in the active site among arginine (Arg 11, 15, 79) and histidine (His 12, 257) residues [2]. To gain better understanding of formation of the hPAPtartrate complex and retention of the enzyme configuration we have characterized the structure and stability of hPAP in the absence and the presence of tartaric acid. For this purpose we used Fourier transform infrared spectroscopy (FTIR) and differential scanning calorimetry (DSC), the techniques that are particularly suitable for studying proteins in solutions [7].

\section{MATERIALS AND METHODS}

Materials. Prostatic acid phosphatase was purified from human seminal plasma using affinity chromatography according to Van Etten \& Saini [3]. Phosphatase concentration was determined spectrophotometrically by using the hPAP absorption coefficient of $\mathrm{A}_{280}^{0.1 \%}=$ 1.44 [8]. Tartaric acid was obtained from Sigma (St. Louis, MO, U.S.A.). All other reagents were of analytical grade obtained from POCH (Poland).

Infrared spectroscopy. The protein solution in $\mathrm{H}_{2} \mathrm{O}$ buffer were placed in a demountable temperature controlled liquid cell (Harrick Scientific, Ossining, NY, U.S.A.) be- tween two $\mathrm{CaF}_{2}$ windows using 6-mm-thick Mylar spacer. FTIR spectra were recorded using a MIDAC FTIR spectrometer (MIDAC Corp., Costa Mesa, CA, U.S.A.) equipped with a DTGS detector. To eliminate spectral contributions of atmospheric water vapour during data acquisition, the spectrometer was continuously purged with dry nitrogen. All spectra were recorded at a resolution of $4 \mathrm{~cm}^{-1}$. One hundred scans were co-added and Fourier-transformed. Spectra of the buffer were acquired under the same scanning and temperature conditions. The protein spectra were obtained by subtracting buffer absorption from spectra of solutions of hPAP. Subtractions of $\mathrm{H}_{2} \mathrm{O}$ absorption from the spectra were based on two criteria: (1) a straight baseline from 2000 to $1725 \mathrm{~cm}^{-1}$; and (2) no lobes with a negative absorbance [9]. The final difference spectrum was smoothed out by a nine-point Savitzky-Golay function [10] to remove a possible white noise. Measurements were performed at protein concentration of 7-16 $\mathrm{mg} / \mathrm{ml}$ in $10 \mathrm{mM}$ Tris/HCl buffer, $\mathrm{pH}$ 7.5. The cell was maintained at a constant temperature of $25^{\circ} \mathrm{C}$, which was measured by iron-constantan thermocouple imbedded in the body of the cell. For experiments, in which the temperature was varied, 25 scans were co-added and Fourier-transformed. Thermal experiments were carried out in the absence and in the presence of $1 \mathrm{mM}$ tartaric acid. The temperature was raised by $5^{\circ} \mathrm{C}$ steps and was controlled at $\pm 1.5^{\circ} \mathrm{C}$ by temperature controller Shimaden SR27 (Shimaden Co., Ltd., Tokyo, Japan). Before spectrum acquisition samples were maintained for $10 \mathrm{~min}$ at the indicated temperature. Curve-fitting was performed using the software Spectra Calc (Galactic Industries Corp., Salem, NH, U.S.A.). The number of bands, peak positions, peak widths and heights were determined from the deconvoluted and second-derivative spectra. Gaussian bands were used [11]. The Fourier self-deconvolution program supplied with Spectra Calc based on the method developed by Griffiths \& Pariente [12] was used with a $\gamma$ 
factor of 2 and an $f$ filter that ranged from 0.3 to 0.35 , where $\gamma$ is the band narrowing factor, and $f$ is a high-pass filter. Savitzky-Golay [10] second derivatives were taken with 11-17 convolution points.

Differential scanning calorimetry. Calorimetric studies were performed at a scanning rate of $1^{\circ} \mathrm{C} / \mathrm{min}$ using an adiabatic capillary scanning microcalorimeter DASM-4 (cell volume $0.4699 \mathrm{ml}$ ), manufactured by the Bureau of Biological Instrumentation of the Academy of Sciences of the U.S.S.R. The protein concentration for calorimetric studies was about $1 \mathrm{mg} / \mathrm{ml}$ in $10 \mathrm{mM}$ Tris/HCl buffer, $\mathrm{pH}$ 7.5. In the DSC experiments with tartaric acid, the ligand was added to the protein solution in $10 \mathrm{mM}$ Tris $/ \mathrm{HCl}, \mathrm{pH} 7.5$, to give the desired concentration of tartaric acid ( $1 \mathrm{mM})$. The reference solution, protein sample, and calorimeter cells were at room temperature at the time of sample introduction and were subsequently cooled to $2^{\circ} \mathrm{C}$. Calorimetric measurements were recorded from 10 to $90^{\circ} \mathrm{C}$ at a heating rate of $1^{\circ} \mathrm{C} / \mathrm{min}$. An external pressure of 1 atm was maintained during all DSC runs to prevent possible degassing of the solution on heating. Buffer-buffer base lines were subtracted from buffer-sample scans. $\Delta \mathrm{H}$ was calculated from the area under each calorimetric peak.

\section{RESULTS}

\section{Effect of temperature on the structure of hPAP}

In order to gain information about the thermal stability of hPAP and about the mechanism of hPAP unfolding at elevated temperature we studied the temperature dependence of hPAP FTIR spectra. Quantitative information on the secondary structure of hPAP can be obtained from an analysis of the amide I band of deconvoluted spectra. We calculated the quantitative contributions of individual component bands to the total amide I contour using curve-fitting of the deconvoluted spectrum. Published FTIR data of proteins in water [7, 13-16] were used to assign the individual bands to secondary structure elements. The amide I band of hPAP is composed of five components at 1691, 1676, 1659, 1642 and $1627 \mathrm{~cm}^{-1}$, and a weaker shoulder at 1607 $\mathrm{cm}^{-1}$ (Fig. 1A - bottom trace). The band at $1607 \mathrm{~cm}^{-1}$ is due to side chain frequencies and was not included in the secondary structure calculations. However, it was necessary to include it for curve-fitting in order to avoid distortions of the amide I band [17]. The strongest band at $1659 \mathrm{~cm}^{-1}$ was assigned to $\alpha$-helical segments [7, 14, 15]. The component at $1691 \mathrm{~cm}^{-1}$ was assigned to $\beta$-antiparallel structure [7, 15, 16]. The component at 1676 $\mathrm{cm}^{-1}$ was interpreted as arising from turn structures [14, 15] and the band $1627 \mathrm{~cm}^{-1}$ was attributed to $\beta$-sheet structure [13-16]. Random conformations are usually associated with a broad band at 1641 and $1646 \mathrm{~cm}^{-1}$ therefore band observed at $1642 \mathrm{~cm}^{-1}$ was assigned to random structures [15, 16].

Figure 1A shows deconvoluted spectra of hPAP at $25^{\circ} \mathrm{C}$ and $55^{\circ} \mathrm{C}$. At $25^{\circ} \mathrm{C}$ the amide I band is rather asymmetric and has a peak maximum at $1659 \mathrm{~cm}^{-1}$ which corresponds to $\alpha$-helix structure. To follow the thermal unfolding of hPAP we used the intensity ratio of the amide $I$ band at $1645 \mathrm{~cm}^{-1}$ and $1610 \mathrm{~cm}^{-1}$ (Fig. 2). The latter band is expected to be invariant with temperature as due to side-chain oscillations. Heating the sample from $25^{\circ} \mathrm{C}$ to $50^{\circ} \mathrm{C}$ had little effect on the infrared spectrum. However, the spectrum at $55^{\circ} \mathrm{C}$ was different, showing a change in the structure located between $50^{\circ} \mathrm{C}$ and $55^{\circ} \mathrm{C}$, which corresponds to thermal denaturation. The midpoint of denaturation occurs at about $52.5^{\circ} \mathrm{C}$ (of Fig. 2). The spectrum recorded at $55^{\circ} \mathrm{C}$ corresponding to the final stage of thermal transition (Fig. 1A, top trace) is distinctly different from that recorded at $25^{\circ} \mathrm{C}$; it has a broad symmetric peak centred around $1645 \mathrm{~cm}^{-1}$, indicative of the predominance of random structure. An increase in the composite band 
corresponding to random structure, slightly shifted in the spectrum at $55^{\circ} \mathrm{C}$ to 1645 from $1642 \mathrm{~cm}^{-1}$ and a decrease of the composite band at $1659 \mathrm{~cm}^{-1}$ indicate an increase in the amount of random structures and a decrease in the $\alpha$-helix content. peratures, up to $80^{\circ} \mathrm{C}$, an increase in the intensity of the $1645 \mathrm{~cm}^{-1}$ amide I band was observed, indicating the beginning of transition due to protein unfolding. The spectrum recorded at $80^{\circ} \mathrm{C}$ (Fig. 1B, top trace) differs significantly from that recorded at $25^{\circ} \mathrm{C}$ and is
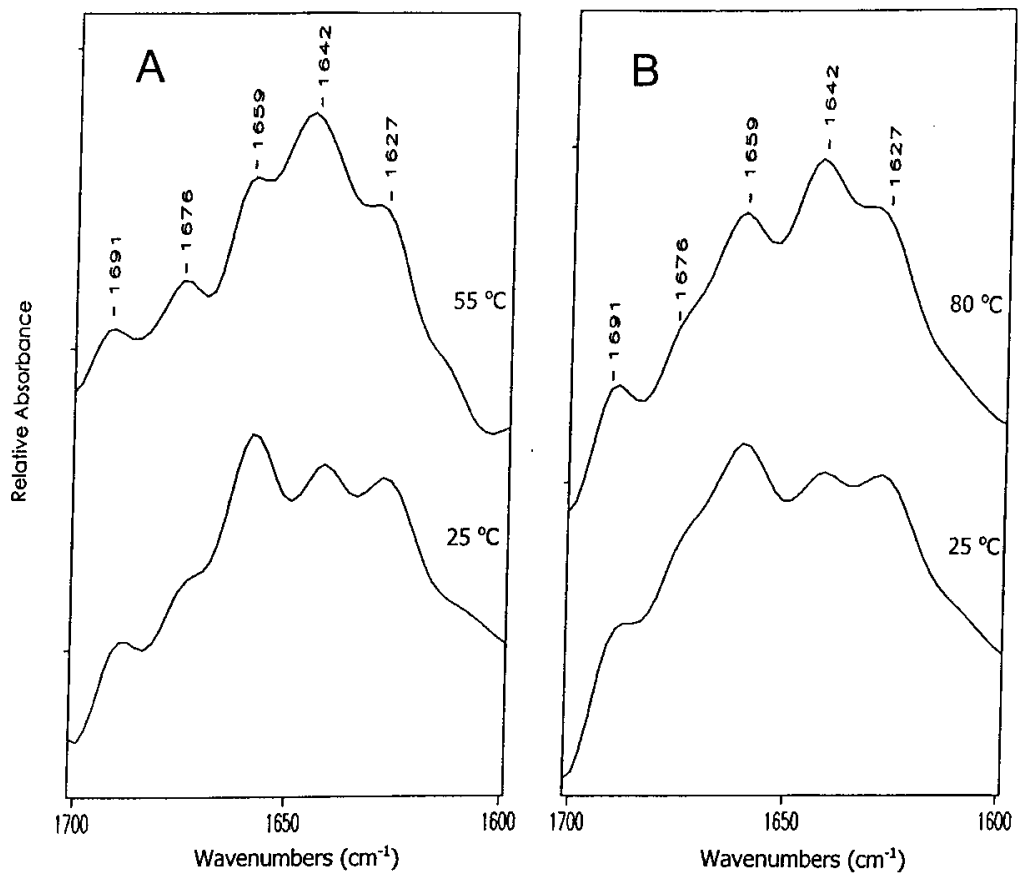

Figure 1. (A) Deconvoluted infrared spectra of hPAP in the absence of tartaric acid in $10 \mathrm{mM}$ Tris/HCl, pH 7.5 , at $25^{\circ} \mathrm{C}$ and $55^{\circ}$ C. (B) Deconvoluted infrared spectra of hPAP in the presence of $1 \mathrm{mM}$ tartaric acid in $10 \mathrm{mM}$ Tris/HCl, pH 7.5 , at $25^{\circ} \mathrm{C}$ and $80^{\circ} \mathrm{C}$.

\section{Effect of tartaric acid on the structure of hPAP}

Experiments with hPAP at different temperatures were carried out in the presence of excess tartaric acid. Figure 1B shows deconvoluted spectra of hPAP in the presence of 1 $\mathrm{mM}$ tartaric acid in $10 \mathrm{mM}$ Tris/ $\mathrm{HCl}, \mathrm{pH} 7.5$, at $25^{\circ} \mathrm{C}$ and $80^{\circ} \mathrm{C}$. A visual comparison of deconvoluted spectra recorded at room temperature in the presence of tartaric acid and in its absence (Fig. 1B and 1A, bottom traces, correspondingly) reveals only minor differences. However, the hPAP spectra recorded in the presence of tartaric acid indicate that ligand binding results in an increase in the thermal stability of the enzyme. The temperature dependence of the intensity ratio of the amide I band at $1645 \mathrm{~cm}^{-1}$ to that at 1610 $\mathrm{cm}^{-1}$ (Fig. 3) shows that the intensity of the amide I band at $1645 \mathrm{~cm}^{-1}$ remained relatively unchanged up to $65^{\circ} \mathrm{C}$. At higher tem- similar to that recorded in the absence of tartaric acid at $55^{\circ} \mathrm{C}$. As it can be judged from the data in Fig. 1B, the midpoint of denaturation for hPAP in the presence of tartaric acid was at about $72^{\circ} \mathrm{C}$. Table 1 lists the secondary structure of hPAP in the absence and in the presence of tartaric acid at different temperatures, obtained using the curve-fitting technique based on peak positions determined from deconvoluted and second derivative spectra. Comparison of the secondary structure content by FTIR for the native and thermally denatured protein in the absence and in the presence of tartaric acid indicates that thermal denaturation results mainly in the loss of the $\alpha$-helix structure. However, the denatured protein in the absence of tartaric acid retains a significant amount of secondary structure in that it has $15 \% \alpha$-helix and $14 \%$ $\beta$-sheet. The lost secondary structure is replaced predominantly by an increase in random structures up to $60 \%$. As seen in Table 1, 
Table 1. Secondary structure analysis of hPAP in the absence and in the presence of tartaric acid

\begin{tabular}{lcccc}
\hline \multirow{2}{*}{ Amide I components } & \multicolumn{3}{c}{ \% of total secondary structure } \\
\cline { 2 - 5 } & hPAP & \multicolumn{3}{c}{ hPAP with tartaric acid } \\
\hline$\alpha$-helix & $25^{\circ} \mathrm{C}$ & $55^{\circ} \mathrm{C}$ & $25^{\circ} \mathrm{C}$ & $80^{\circ} \mathrm{C}$ \\
$\beta$-sheet & 34 & 15 & 37 & 15 \\
random & 15 & 14 & 24 & 21 \\
other & 29 & 60 & 16 & 33 \\
\hline
\end{tabular}

in the presence of tartaric acid the increase in temperature to $80^{\circ} \mathrm{C}$ leads to a decrease of the $\alpha$-helix structure content to $15 \%$ and of the $\beta$-sheet structure to $21 \%$ and to a raise of random conformations up to $33 \%$.

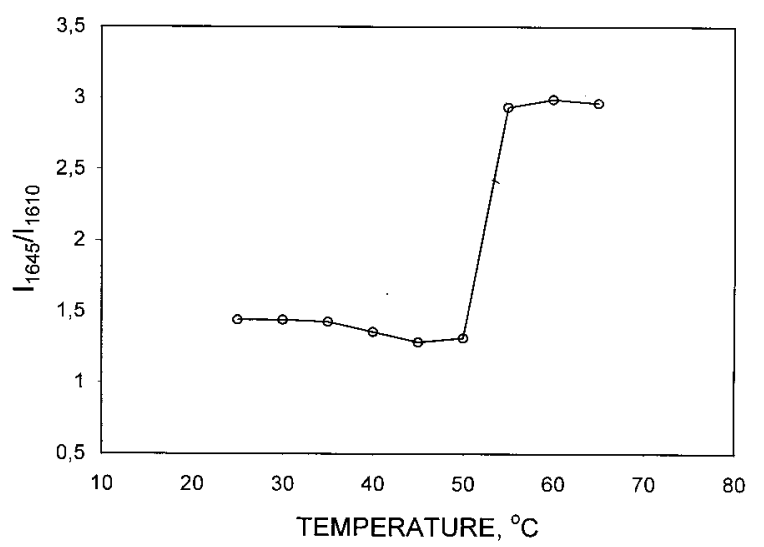

Figure 2. Temperature dependence of the ratio of the amide I band intensity at $1645 \mathrm{~cm}^{-1}$ to that at $1610 \mathrm{~cm}^{-1}$ for hPAP in the absence of tartaric acid in $10 \mathrm{mM}$ Tris/HCl, pH 7.5 .

\section{Differential scanning calorimetry}

Further information about binding of tartaric acid and thermal stability of hPAP was obtained using differential scanning calorimetry. Typical DSC scans of hPAP recorded in the absence and in the presence of L(+)-tartaric acid are shown in Fig. 4. Both samples show an endothermic thermal transition. The DSC scan of hPAP in the buffer (Fig. 4A) shows the endothermic transition with a midtransition temperature of $49.5^{\circ} \mathrm{C}$ and with a calorimetric enthalpy of $895.6 \mathrm{kcal} / \mathrm{mol}$. The temperature of $49.5^{\circ} \mathrm{C}$ is in agreement with the temperature of the unfolding determined by FTIR spectroscopy (Fig. 2). The thermal effect corresponds to $37.5 \mathrm{~J} / \mathrm{g}$, and is in accordance to protein denaturation values ranging from 25 to $50 \mathrm{~J} / \mathrm{g}$ [18]. Upon binding of tartaric acid to hPAP, the DSC scan of the

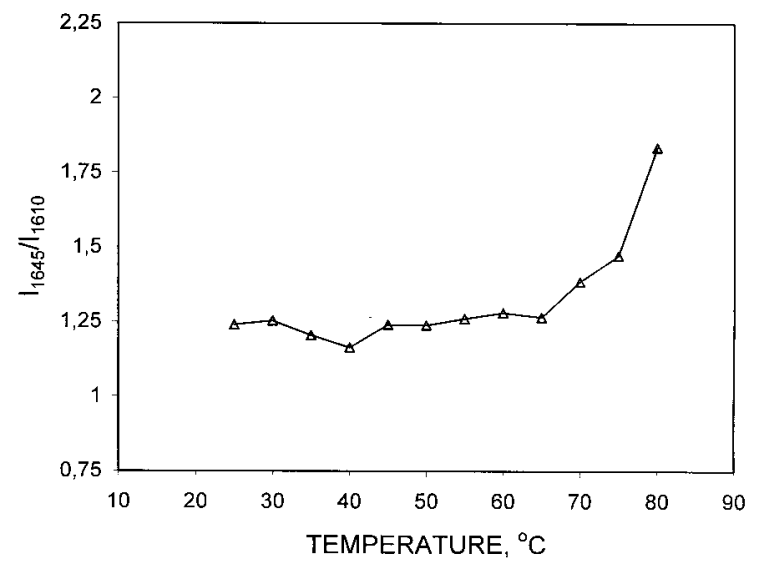

Figure 3. Temperature dependence of the ratio of the amide $I$ band intensity at $1645 \mathrm{~cm}^{-1}$ to that at $1610 \mathrm{~cm}^{-1}$ for hPAP in the presence of tartaric acid in $10 \mathrm{mM}$ Tris/HCl, pH 7.5 .

complexed enzyme shows an endothermic transition with a midtransition temperature of $68.5^{\circ} \mathrm{C}$ (Fig. 4B). This confirms the result obtained by FTIR spectroscopy that hPAP in the presence of tartaric acid becomes denatured at higher temperatures. The calorimetric enthalpy of the transition is $1107.8 \mathrm{kcal} /$ mol (the thermal effect corresponding to 46.4 $\mathrm{J} / \mathrm{g}$ ). The value for $\Delta \mathrm{H}$ is larger in the presence of the ligand. Both DSC curves show a distinct asymmetry, being less steep on the low-temperature side than on the high-temperature side. Such asymmetry could be due to intermediate steps between the native and 
denatured states [19]. To a similar suggestion lead the results of the work of Kuciel et al. [20] on reversible denaturation of $\mathrm{hPAP}$, in which a model with a dimeric intermediate was presented.
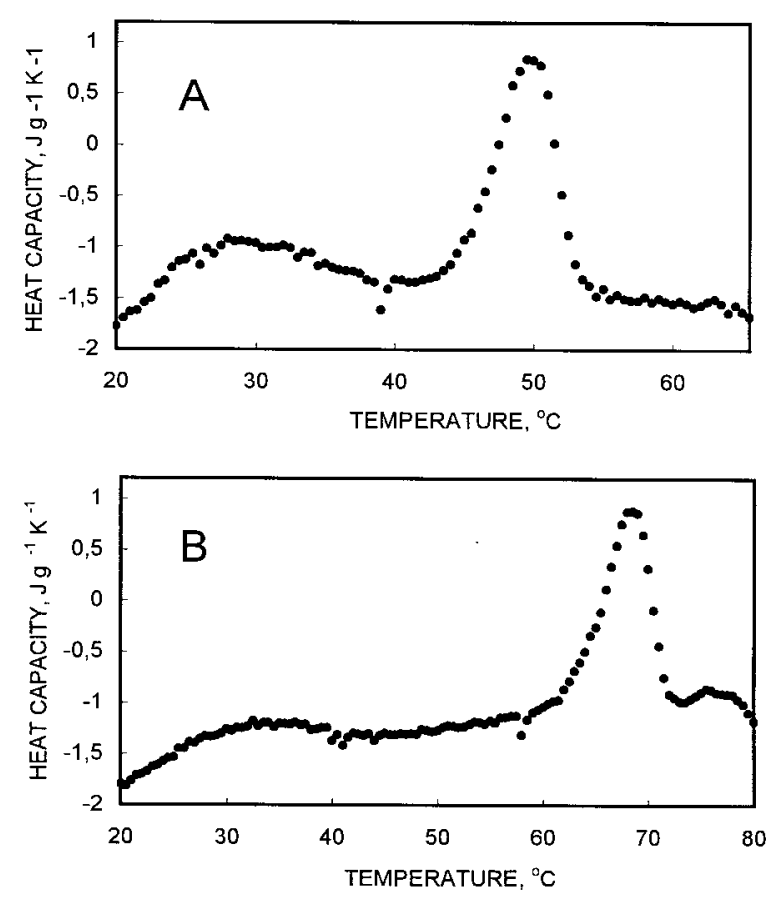

Figure 4. DSC scans of hPAP in $10 \mathrm{mM}$ Tris/ $\mathrm{HCl}$, pH 7.5, in the absence (A) and in the presence of 1 mM tartaric acid (B).

\section{DISCUSSION}

The structure of hPAP consists of two glycosylated identical subunits with the same apparent molecular mass of $50 \mathrm{kDa}$ [21]. The subunits are linked by noncovalent bonds, and denaturation-renaturation experiments have shown the importance of the dimer formation for the catalytic activity [20, 22]. X-Ray crystallography showed that each subunit has two domains; the larger one is of $\alpha / \beta$ type and the smaller - an $\alpha$ helix [4]. The enzyme undergoes a major conformational transition in the temperature range between about 50 and $60^{\circ} \mathrm{C}$ at $\mathrm{pH} 2.5$, when it loses its catalytic activity and native conformation as observed by spectrofluorometric methods [22] and CD spectra [20, 23]. Analyses of circular dichroism spectra of hPAP, especially the band at $210-230 \mathrm{~nm}$, indicated that the native protein contains a substantial amount of $\alpha$-helical structure, which, upon denaturation in $6 \mathrm{M}$ urea at $\mathrm{pH} 2.5$, became largely unfolded [20]. The denatured dimeric enzyme was completely dissociated into monomers and lost its catalytic activity. The decrease in the $\alpha$-helix content of denatured hPAP led us to carry out a detailed examination of the enzyme structure and stability. IR spectroscopy reports directly on the nature of hydrogen bonding involving $\mathrm{C}=\mathrm{O}$ and $\mathrm{NH}$ moieties and has proven sensitive to even subtle changes in the secondary structure of the protein backbone [24, 25]. Qualitative and quantitative analyses of the amide I components of hPAP provided more information on changes in structure of the thermally denatured enzyme and on stabilization of its native structure in the presence of the competitive inhibitor L(+)-tartrate. The secondary structure of native hPAP at $\mathrm{pH} 7.5$ and $25^{\circ} \mathrm{C}$ was resolved into its structural motifs, and quantitative analysis of the amide I components performed by a curve-fitting procedure revealed $34 \% \alpha$-helix and $15 \% \beta$-sheet. X-Ray crystallographic values for $\alpha$-helix and $\beta$-sheet are $42 \%$ and $14 \%$ [4], which is in reasonable agreement with our FTIR data. These FTIR data suggest that the secondary structure of hPAP is not significantly altered by binding of tartaric acid. This is in agreement with X-ray studies [2, 5] which show that hPAP, after tartrate ions binding, retains most of its secondary structure. X-Ray data indicate that changes occur mainly in the active site at arginine and histidine residues, involving positional shifts of the side groups to accommodate optimal hydrogen binding to inhibitor and involve a variation in the tertiary rather than in the secondary structure [2]. The conformational change that occurs upon tartaric acid binding is primarily reflected in an increase in thermal stability of the enzyme. Our FTIR and DSC results show that binding of tartaric acid leads to thermal stabilization of the enzyme by almost $20^{\circ} \mathrm{C}$. Though the conformational changes, mainly connected 
with extensive hydrogen bonding between hydroxyl group of the tartrate ion and the enzyme molecule, are small they are sufficient for thermal stabilization of the enzyme. IR spectroscopy and scanning calorimetry consistently indicate that, in the absence of tartrate, hPAP undergoes a conformational transition with the midpoint between $49.5^{\circ} \mathrm{C}$ and $52.5^{\circ} \mathrm{C}$ depending on the detection method. From our kinetic studies [26] it is evident that hPAP at $\mathrm{pH} 7.0$ and $55^{\circ} \mathrm{C}$ completely loses its catalytic activity and dissociates into two subunits. The FTIR show that after the thermal denaturation hPAP retains some of the secondary structure, having $15 \% \alpha$-helix and $14 \% \beta$-sheet. Tartaric acid acts as a protector of the secondary structure and, in its presence, the enzyme becomes unfolded to a smaller extent, having only $33 \%$ of random structure. Temperature induced changes in the conformation of hPAP in the absence and in the presence of tartaric acid helped to better understand the mechanism of unfolding of this enzyme and could be useful in further investigations of the biological function of hPAP.

We thank Drs. R. Kuciel and A. Mazurkiewicz for supplying the enzyme, and Professor Wojciech Zielenkiewicz and Dr. Anna Zielenkiewicz from Institute of Physical Chemistry of Polish Academy of Sciences for help in calorimetric experiments.

\section{R E F E R E N C E S}

1. Kilsheimer, G.S. \& Axelrod, B. (1957) Inhibition of prostatic acid phosphatase by $\alpha$-hydroxy carboxylic acids. J. Biol. Chem. 227, 879-890.

2. La Count, M.W., Handy, G. \& Lebioda, L. (1998) Structural origins of L(+)tartrate inhibition of human prostatic acid phosphatase. $J$. Biol. Chem. 273, 30406-30409.
3. Van Etten, R.L. \& Saini, M.S. (1978) Selective purification of tartrate inhibitable acid phosphatase: Rapid and efficient purification of human and canine prostatic acid phosphatase. Clin. Chem. 24, 1525-1530.

4. Jacob, C.G., Lewinski, K., Kuciel, R., Ostrowski, W. \& Lebioda, L. (2000) Crystal structure of human prostatic acid phosphatase. The Prostate 42, 211-218.

5. Lovelace, L., Lewinski, K., Jakob, C.G., Kuciel, R., Ostrowski, W. \& Lebioda, L. (1997) Prostatic acid phosphatase: Structural aspects of inhibition by L(+)-tartrate ions. Acta Biochim. Polon. 44, 673-678.

6. Kuciel, R., Jakob, C.G., Lebioda, L. \& Ostrowski, W.S. (1992) Crystallization of human prostatic acid phosphatase using biphasic systems. J. Crystal Growth 122, 199-203.

7. Hadden, J.M., Bloemendal, M., Haris, P.I., Srai, S.K.S. \& Chapman, D. (1994) Fourier transform infrared spectroscopy and differential scanning calorimetry of transferrins: $\mathrm{Hu}$ man serum transferrin, rabbit serum transferrin and human lactoferrin. Biochim. Biophys. Acta 1205, 59-67.

8. Bobrzecka, K., Ostrowski, W. \& Rybarska, J. (1968) The effect of iodination on the activity and structure of acid phosphomonoesterase from human prostate. Acta Biochim. Polon. 15, 369-379.

9. Olinger, J.M., Hill, D.M., Jakobsen, R.J. \& Brody, R.S. (1986) Fourier transform infrared studies of ribonuclease in $\mathrm{H}_{2} \mathrm{O}$ and ${ }^{2} \mathrm{H}_{2} \mathrm{O}$ solutions. Biochim. Biophys. Acta 869, 89-98.

10. Savitzky, A. \& Golay, J.E. (1964) Smoothing and differentiation of data by simplified least squares procedures. Anal. Chem. 36, 16271639 .

11. Byler, D.M. \& Susi, H. (1986) Examination of the secondary structure of proteins by deconvoluted FTIR spectra. Biopolymers 25, 469487. 
12. Griffiths, P.R. \& Pariente, G.L. (1986) Introduction to spectral deconvolution. Trends Anal. Chem. 5, 209-215.

13. Dong, A., Huang, P. \& Caughey, W.S. (1990) Protein secondary structures in water from second-derivative amide I infrared spectra. Biochemistry 29, 3303-3308.

14. Fabian, H., Naumann, D., Misselwitz, R., Ristau, O., Gerlach, D. \& Welfle, H. (1992) Secondary structure of streptokinase in aqueous solution: A Fourier transform infrared spectroscopic study. Biochemistry 31, 6532-6538.

15. Ahmed, A., Tajmir-Riahi, H.A. \& Carpentier, R. (1995) A quantitative secondary structure analysis of the $33 \mathrm{kDa}$ extrinsic polypeptide of photosystem II by FTIR spectroscopy. FEBS Lett. 363, 65-68.

16. Bramanti, E. \& Benedetti, E. (1996) Determination of the secondary structure of isomeric forms of human serum albumin by a particular frequency deconvolution procedure applied to Fourier transform IR analysis. Biopolymers 38, 639-653.

17. Casal, H.L., Kohler, U. \& Mantsch, H.H. (1988) Structural and conformational changes of $\beta$-lactoglobulin B: An infrared spectroscopic study of the effect of $\mathrm{pH}$ and temperature. Biochim. Biophys. Acta 957, 11-20.

18. Privalov, P.L. (1982) Stability of proteins. Proteins which do not present a single cooperative system. Adv. Protein Chem. 35, 1-104.

19. Hu, C.Q. \& Sturtevant, J.M. (1987) Thermodynamic study of yeast phosphoglycerate kinase. Biochemistry 26, 178-182.
20. Kuciel, R., Mazurkiewicz, A. \& Ostrowski, W.S. (1996) The folding intermediate of reversibly denatured human prostatic acid phosphatase. Intern. J. Biol. Macromol. 18, 167175.

21. Luchter-Wasyl, E. \& Ostrowski, W. (1974) Subunit structure of human prostatic acid phosphatase. Biochim. Biophys. Acta 365, 349-359.

22. Ostrowski, W.S., Kuciel, R., Tanaka, F. \& Yagi, K. (1993) Fluorometric analysis of native, urea-denatured and refolded human prostatic acid phosphatase. Biochim. Biophys. Acta 1164, 319-326.

23. Ostrowski, W., Bhargava, A.K., Dziembor, E., Gizler, M., Gryszkiewicz, J. \& Barnard, E.A. (1976) Acid phosphomonoesterase of human prostate. Carbohydrate content and optical properties. Biochim. Biophys. Acta 453, 262-269.

24. Surewicz, W.K., Mantsch, H.H. \& Chapman, D. (1993) Determination of protein secondary structure by Fourier transform infrared spectroscopy: A critical assessment. Biochemistry 32, 389-394.

25. Surewicz, W.K. \& Olesen, P.R. (1995) On the thermal stability of $\alpha$-crystallin: A new insight from infrared spectroscopy. Biochemistry 34, 9655-9660.

26. Ostrowski, W.S. (1988) Acid Phosphatase of Human Prostate Gland. Ossolineum, Wrocław (in Polish). 